\title{
REMARKS ON A RESULT OF HAYMAN
}

\author{
By BAO-QIN LI
}

\section{Introduction.}

In this paper, we use the usual notation of Nevanlinna theory ${ }^{[3]}$.

Suppose that $f(z)=\sum_{n=0}^{\infty} a_{n} z^{\lambda_{n}}$ is a transcendental entire function, where $a_{n} \neq 0$ $(n=0,1,2, \cdots)$ and $\left\{\lambda_{n}\right\}$ is arranged in increasing order. Also let $g(z)$ be an arbitrary entire function growing slowly compared with the function $f(z)$, i.e., $T(r, g)=o\{T(r, f)\}$ as $r \rightarrow \infty$. Following Hayman ${ }^{[4]}$, if $f(z)$ has finite order, we define

$$
\delta_{S}(g(z), f)=1-\lim _{r \rightarrow \infty} \frac{N\left(r, \frac{1}{f-g(z)}\right)}{T(r, f)} .
$$

If $f(z)$ has infinite order, let $E$ be any set in $(1, \infty)$ having finite length. We define

$$
\delta_{S}(g(z), f)=1-\sup _{E} \lim _{r \rightarrow \infty, r \notin E} \frac{N\left(r, \frac{1}{f-g(z)}\right)}{T(r, f)}=\inf _{E} \varlimsup_{r \rightarrow \infty, r \notin E} \frac{m\left(r, \frac{1}{f-g(z)}\right)}{T(r, f)} .
$$

Obviously,

$$
\delta(g(z), f)=1-\varlimsup_{r \rightarrow \infty} \frac{N\left(r, \frac{1}{f-g(z)}\right)}{T(r, f)} \leqq \delta_{S}(g(z), f) .
$$

In particular, when $g(z) \equiv a$ ( $a$ is a constant) we get the definition of $\delta_{S}(a, f)$ defined by Hayman ${ }^{[4]}$.

Under the above definitions, Hayman ${ }^{[4]}$ proved

THEOREM A. Let $d_{n}$ be the highest common factor of all the numbers $\lambda_{m+1}$ $-\lambda_{m}$ for $m \geqq n$ and suppose that

$$
d_{n} \longrightarrow \infty \text { as } n \rightarrow \infty \text {. }
$$

Then $\delta_{S}(a, f)=0$ for every finite complex number $a$.

With the hypotheses of Theorem A, we proved in $[2] \Theta_{S}(g(z), f) \leqq 1 / 2$ for every function $g(z)$ satisfying $T(r, g)=o\{T(r, f)\}$. Now we further prove

Received July 15, 1987 
$\delta_{S}(g(z), f)=0$ for every entire function $g(z)$ satisfying $T(r, g)=o\{T(r, f)\}$ as $r \rightarrow \infty$. That is, we shall prove

THEOREM 1. Let $d_{n}$ be the highest common factor of all the numbers $\lambda_{m+1}$ $-\lambda_{m}$ for $m \geqq n$ and suppose that

$$
d_{n} \longrightarrow \infty \quad \text { as } n \rightarrow \infty \text {. }
$$

Then $\delta_{S}(g(z), f)=0$ for every entire function $g(z)$ satisfying $T(r, g)=o\{T(r, f)\}$ as $r \rightarrow \infty$.

Clearly, Theorem 1 is an extension of Theorem A.

\section{Proof of Theorem 1 .}

From now on we denote by $S(r, f)$ any term which satisfies $S(r, f)=$ $o\{T(r, f)\}$ as $r \rightarrow \infty$ outside some set of finite length. In particular, if $f(z)$ is of finite order, then we assume that $S(r, f)=o\{(T(r, f))\}$ as $r \rightarrow \infty$ without exceptional set. Also suppose that $a$ and $b$ are two positive numbers. " $a \mid b$ " implies that there exists a positive integer $n$ such that $b=n \cdot a$.

In order to obtain Theorem 1 , we need the following lemma of [1].

LEMMA 1. If $F(z)$ is an entire function and $g_{1}(z), g_{2}(z), \cdots, g_{m}(z)$ are distinct entire functions satisfying $T\left(r, g_{j}\right)=o\{T(r, F)\}$ as $r \rightarrow \infty(j=1,2, \cdots, m)$, then

$$
\sum_{j=1}^{m} m\left(r, \frac{1}{F-g_{j}(z)}\right) \leqq T(r, F)+S(r, F) .
$$

Proof of Theorem 1. Suppose that $g(z)$ is any entire function satisfying

$$
T(r, g)=o\{T(r, f)\} \quad \text { as } \quad r \rightarrow \infty .
$$

We then discuss two cases separately.

Case (A). $g(z)=\sum_{v=0}^{t} b_{v} z^{h_{v}}$ is a polynomial.

We write

$$
f(z)-g(z)=\sum_{v=0}^{\infty} \eta_{v} z^{\beta v},
$$

where $\eta_{v} \neq 0$ and $\left\{\beta_{v}\right\}$ is arranged in increasing order. Let $d_{n}^{*}$ be the highest common factor of all the numbers $\beta_{m+1}-\beta_{m}$ for $m \geqq n$. Obviously we have $d_{n}^{*} \rightarrow \infty$ as $n \rightarrow \infty$. Hence by Theorem A we have, in view of (1),

$$
\delta_{S}(g(z), f)=\delta_{S}(0, f-g)=0 .
$$

Case (B). $\quad g(z)=\sum_{v=0}^{\infty} b_{v} z^{h_{v}}$ is transcendental, where $b_{v} \neq 0(v=0,1,2, \cdots)$ and 
$\left\{h_{v}\right\}$ is arranged in increasing order.

Suppose contrary to hypothesis that $\delta_{S}(g(z), f)>0$ and choose a positive integer $T$ such that

$$
\delta_{S}(g(z), f)>\frac{1}{T}
$$

We write

$$
f(z)-g(z)=\sum_{v=0}^{\infty} \eta_{v} z^{\beta},
$$

where $\eta_{v} \neq 0(v=0,1,2, \cdots)$ and $\left\{\beta_{v}\right\}$ is arranged in increasing order.

Let us choose an integer $n$ so large that

$$
n>T+2 \text { and } d_{n}>T ! T \beta_{T+2} \text {. }
$$

We assume $h_{v_{n}}$ as the minimum of numbers $h_{v}(v=0,1,2, \cdots)$ satisfying $h_{v} \geqq \lambda_{n}$. Again we consider two subcases separately.

Case (B.1). There exists an integer $v_{N}>v_{n}$ such that

$$
\left(h_{v_{N}}-\lambda_{n}, d_{n}\right)<\frac{d_{n}}{T} \text {. }
$$

In this case we write

$$
f(z)-g(z)=\sum_{v=0}^{\infty} a_{v} z^{\lambda_{v}}-\sum_{v=0}^{\infty} b_{v} z^{h_{v}}=\phi_{0}(z)+\phi_{1}(z),
$$

say, where $\phi_{0}=\sum_{v=0}^{n-1} a_{v} z^{\lambda v}-\sum_{v=0}^{\infty} b_{v} z^{h_{v}}, \phi_{1}=\sum_{v=n}^{\infty} a_{v} z^{\lambda v}$.

By $d_{n} \mid\left(\lambda_{m+1}-\lambda_{m}\right)$ for $m \geqq n$, we have

$$
d_{n} \mid\left(\lambda_{v}-\lambda_{n}\right) \quad \text { for } \quad v \geqq n+1 .
$$

We set $\omega_{n}=\exp \left(2 \pi i / d_{n}\right)$. Then for each non-negative integer $j$ we have

$$
\begin{aligned}
& \phi_{1}\left(\omega_{n}^{j} z\right)=\omega_{n}^{j \lambda_{n}} \phi_{1}(z), \\
& \begin{aligned}
\phi_{1}\left(\omega_{n}^{j} z\right)+\phi_{0}\left(\omega_{n}^{j} z\right) & =\omega_{n}^{j \lambda_{n}}\left\{\phi_{1}(z)+\omega_{n}^{-j \lambda}{ }^{j} \phi_{0}\left(\omega_{n}^{j} z\right)\right\} \\
& =\omega_{n}^{j \lambda_{n}}\left\{\phi_{1}(z)-Q_{j}(z)\right\},
\end{aligned}
\end{aligned}
$$

say, where $Q_{j}(z)=-\omega_{n}^{-j \lambda} \phi_{0}\left(\omega_{n}^{j} z\right)$. We deduce that

$$
m\left(r, \frac{1}{\phi_{1}(z)-Q_{j}(z)}\right)=m\left(r, \frac{1}{\phi_{1}(z)+\phi_{0}(z)}\right)=m\left(r, \frac{1}{f-g(z)}\right) .
$$

Also according to (1) we have

$$
T\left(r, Q_{j}(z)\right)=o\left\{T\left(r, \phi_{1}\right)\right\} \quad \text { as } r \rightarrow \infty .
$$

Obviously, $h_{v_{N}}>h_{v_{n}} \geqq \lambda_{n}$. Thus the coefficient of $z^{h \cdot v_{N}}$ in $Q_{j}(z)$ is 


$$
I_{\jmath}=b_{v_{N}} \omega_{n}^{-j \lambda} \omega_{n}^{j h_{v_{N}}}=b_{v_{N}} \omega_{n}^{j\left(h_{v_{N}}-\lambda_{n}\right)}=b_{v_{N}} \exp \left(2 \pi i j\left(h_{v_{N}}-\lambda_{n}\right) / d_{n}\right) .
$$

By (5), the number of distinct values of $I_{\jmath}$ is precisely

$$
d_{n} /\left(h_{v_{N}}-\lambda_{n}, d_{n}\right)>T .
$$

Thus more than $T$ of $Q_{j}(z)$ are distinct from each other. Using (6), (7) and Lemma 1 we deduce that

Hence

$$
\begin{aligned}
(T+1) m\left(r, \frac{1}{f-g(z)}\right) & \leqq T\left(r, \phi_{1}\right)+S\left(r, \phi_{1}\right) \\
& \leqq T(r, f)+S(r, f) \quad(r \rightarrow \infty) .
\end{aligned}
$$

$$
\delta_{S}(g(z), f) \leqq \frac{1}{T+1}<\frac{1}{T}
$$

This contradicts (2).

Case (B.2). For all $v>V_{n}$ we have $\left(h_{v}-\lambda_{n}, d_{n}\right) \geqq d_{n} / T$.

In this case we have

$$
\left(h_{v}-\lambda_{n}, d_{n}\right)=\frac{d_{n}}{c_{v}}
$$

where $c_{v}$ is an integer and $1 \leqq c_{v} \leqq T$.

Clearly $d_{n} / T ! \mid d_{n} / c_{v}$. Thus

$$
\frac{d_{n}}{T !} \mid d_{n} \text { and } \quad \frac{d_{n}}{T !} \mid\left(h_{v}-\lambda_{n}\right) \quad \text { for } \quad v>v_{n} .
$$

By (3)

$$
f(z)-g(z)=\sum_{v=0}^{\infty} a_{v} z^{\lambda}-\sum_{v=0}^{\infty} b_{v} z^{h_{v}}=\sum_{v=0}^{\infty} \eta_{v} z^{\beta} .
$$

Thus we easily deduce, in view of (8) and the definition of $d_{n}$, that there exists an integer $v_{M}$ such that $v_{M} \geqq n$ and

$$
\frac{d_{n}}{T !} \mid\left(\beta_{v}-\beta_{v_{M}}\right) \quad \text { for } \quad v \geqq v_{M}+1 \text {. }
$$

Obviously we have

$$
f(z)-g(z)=\sum_{v=0}^{v_{M}-1} \eta_{v} z^{\beta_{v}}+\sum_{v=v_{M}}^{\infty} \eta_{v} z^{\beta_{v}}=\phi_{0}(z)+\phi_{1}(z),
$$

say, where $\phi_{0}=\sum_{v=0}^{v_{M}-1} \eta_{v} z^{\beta_{v}}$ and $\phi_{1}=\sum_{v=v_{M}}^{\infty} \eta_{v} z^{\beta_{v}}$.

We set $\zeta_{n}=\exp \left(2 \pi i T ! / d_{n}\right)$. Noticing (9), we deduce that

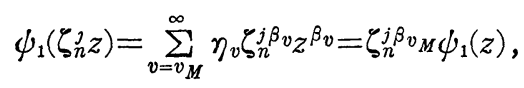

where $j$ is a non-negative integer. 
Hence

$$
\begin{aligned}
\phi_{1}\left(\zeta_{n}^{j} z\right)+\phi_{0}\left(\zeta_{n}^{j} z\right) & =\zeta_{n}^{j \beta} v_{M}\left\{\phi_{1}(z)+\zeta_{n}^{-j \beta v_{M}} \phi_{0}\left(\zeta_{n}^{j} z\right)\right\} \\
& =\zeta_{n}^{j \beta_{v_{M}}}\left\{\phi_{1}(z)-R_{j}(z)\right\},
\end{aligned}
$$

say, where $R_{j}(z)=-\zeta_{n}^{-j \beta_{v}} \psi_{0}\left(\zeta_{n}^{j} z\right)$.

We easily see that

$$
\begin{aligned}
& T\left(r, R_{j}(z)\right)=o\left\{T\left(r, \phi_{1}\right)\right\} \\
& m\left(r, \frac{1}{\psi_{1}-R_{j}(z)}\right)=m\left(r, \frac{1}{\phi_{0}+\phi_{1}}\right)=m\left(r, \frac{1}{f-g(z)}\right) .
\end{aligned}
$$

Now we set

$$
\Delta_{v}=\left(T !\left(\beta_{v_{M}}-\beta_{v}\right), d_{n}\right) \quad(v=2,3, \cdots, T+2) .
$$

Then there must exist an integer $p$ such that $2 \leqq p \leqq T+2$ and

$$
\Delta_{p}=\left(T !\left(\beta_{v_{M}}-\beta_{p}\right), d_{n}\right)<\frac{d_{n}}{T} .
$$

In fact if $\Delta_{v} \geqq d_{n} / T$ for each $v(v=2,3, \cdots, T+2)$, then $\Delta_{v}=d_{n} / q_{v}$ where $q_{v}$ is an integer and $1 \leqq q_{v} \leqq T$. Thus there can be at most $T$ different values of $q_{v}$ and so of $\Delta_{v}$. But $\Delta_{v}(v=2,3, \cdots, T+2)$ must all be distinct (If $\Delta_{u}=\Delta_{v}=m$ for $2 \leqq u<v \leqq T+2$, then $m \mid T !\left(\beta_{v}-\beta_{u}\right)$, which is impossible since by (4) we have $\left.0<T !\left(\beta_{v}-\beta_{u}\right)<T ! \beta_{T+2}<d_{n} / T \leqq m\right)$. Therefore the number of distinct values of $\Delta_{v}(v=2,3, \cdots, T+2)$ is precisely $T+1$. This is a contradiction, which shows that (12) is valid.

Now let us recall the definition of $v_{M}$ and $p$ and notice (4). Clearly we have

$$
\beta_{p} \leqq \beta_{T+2}<\beta_{n} \leqq \beta_{v_{M}} .
$$

Hence the coefficient of $z^{\beta p}$ in $R_{j}(z)$ is

$$
\begin{aligned}
& L_{j}=-\zeta_{n}^{-j \beta} v_{M} \eta_{p} \zeta_{n}^{j \beta} p
\end{aligned}
$$

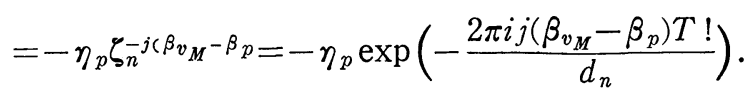

By (12), the number of distinct values of $L$, is precisely

$$
\frac{d_{n}}{\left(T !\left(\beta_{v_{M}}-\beta_{p}\right), d_{n}\right)}>T \text {. }
$$

Thus more than $T$ of the functions $R_{j}(z)$ are distinct from each other. Using (10), (11) and Lemma 1, we obtain that

$$
\begin{aligned}
(T+1) m\left(r, \frac{1}{f-g(z)}\right) & \leqq T\left(r, \phi_{1}\right)+S\left(r, \phi_{1}\right) \\
& \leqq T(r, f)+S(r, f) \quad(r \rightarrow \infty)
\end{aligned}
$$


Therefore

$$
\delta_{S}(g(z), f) \leqq \frac{1}{T+1} \leqq \frac{1}{T} .
$$

This contradicts (2).

According to the above discussion, we deduce that in case (B)

$$
\delta_{S}(g(z), f)=0
$$

This completes the proof of Theorem 1 .

\section{REFERENCES}

[1] C. T. Chuang, Une généralisation d'une inégalité de Nevanlinna, Sci. Sinica, 13 (1964), 887-895.

[2] B.-Q. LI AND C.-J. DAI, A.P. gaps of (b, d) type and modular distribution, Journal of East China Nomal Uuiversity. (to appear).

[3] W.K. Hayman, Meromorphic functions (Oxford University Press, Oxford, 1964)

[4] W.K. Hayman, Value distribution and A.P. gaps, J. London Math. Soc. (2), 28 (1983), 327-338.

Department of Mathematics

Yang Zhou Teachers College

CHINA 